\title{
Response Variability of Linear Stochastic Systems: A General Solution Using Random Matrix Theory
}

\author{
S. Adhikari $^{*}$ \\ Swansea University, Swansea, United Kingdom
}

\begin{abstract}
Uncertainties need to be taken into account for credible predictions of the dynamic response of complex structural systems. Such uncertainties should include uncertainties in the system parameters and those arising due to the modelling of a complex system. In spite of extensive research over the past four decades a general purpose probabilistic predictive code for real-life structural dynamical systems is still not available. The reasons behind this include: (a) the computational time can be prohibitively high compared to a deterministic analysis, and (b) the detailed and complete information regarding parametric and model uncertainties are in general not available. In this paper a Wishart random matrix based approach is proposed to address the above two issues. Closed-form approximate analytical expressions are developed to obtain the mean and covariance of the amplitude of the frequency response function. The method utilizes analytical inversion of the random dynamic stiffness matrix in the frequency domain. The method is applied to vibration of a cantilever plate with uncertainties. The dynamic response obtained using the Wishart random matrix model agree well with the results obtained from the stochastic finite element method.
\end{abstract}

\section{Nomenclature}

$\mathbf{f}(t)$

$\mathbf{M}, \mathbf{C}$ and $\mathbf{K}$

forcing vector

$\mathbf{q}(t)$

mass, damping and stiffness matrices respectively

$\Gamma_{n}(a)$

response vector

$n$

multivariate gamma function

number of degrees of freedom

$p, \boldsymbol{\Sigma}$

scalar and matrix parameters of the Wishart distribution

Conventions

$\begin{array}{ll}(\bullet)^{T} & \text { matrix transposition } \\ |\bullet| & \text { absolute of a complex quantity } \\ \mathbb{R} & \text { space of real numbers } \\ \mathbb{R}_{n}^{+} & \text {space } n \times n \text { real positive definite matrices } \\ \mathbb{R}^{n \times m} & \text { space } n \times m \text { real matrices } \\ \operatorname{det}\{\bullet\} & \text { determinant of a matrix }\end{array}$

${ }^{*}$ Professor of Aerospace Engineering, School of Engineering, Swansea University, Singleton Park, Swansea SA2 8PP, UK, AIAA Senior Member.

Copyright (C) 2008 by the American Institute of Aeronautics and Astronautics, Inc. The U.S. Government has a royalty-free license to exercise all rights under the copyright claimed herein for Governmental purposes. All other rights are reserved by the copyright owner.

\section{1 of 15}

American Institute of Aeronautics and Astronautics 


$\begin{array}{ll}\text { etr }\{\bullet\} & \text { exp }\{\text { Trace }(\bullet)\} \\ \mathrm{E}[\bullet] & \text { expectation operator } \\ \|\bullet\|_{\mathrm{F}} & \text { Frobenius norm of a matrix, }\|\bullet\|_{\mathrm{F}}=\left(\operatorname{Trace}\left((\bullet)(\bullet)^{T}\right)\right)^{1 / 2} \\ \otimes & \text { Kronecker product }\left(\sec ^{1}\right) \\ \sim & \text { distributed as } \\ \text { Trace }(\bullet) & \text { sum of the diagonal elements of a matrix }\end{array}$

\section{Introduction}

Numerical computer codes implementing physics based models are the backbone of today's dynamic analysis of complex systems. Laboratory based controlled tests are often performed to gain insight into some specific physics of a problem. Such tests can indeed lead to new physical laws improving a part of the overall science model. Test data can also be used to calibrate a known model. However, neither of these activities are enough to produce a credible numerical tool because of several types of uncertainties which exist in the whole process of physics based computational predictions. Such uncertainties include, but not limited to (a) parametric uncertainty (e.g, uncertainty in geometric parameters, friction coefficient, strength of the materials involved); (b) model inadequacy (arising from the lack of scientific knowledge about the model which is a-priori unknown); (c) experimental error (uncertain and unknown error percolate into the model when they are calibrated against experimental results); (d) computational uncertainty (e.g, machine precession, error tolerance and the so called ' $h$ ' and 'p' refinements in finite element analysis) and (e) model uncertainty (genuine randomness in the model such as uncertainty in the position and velocity in quantum mechanics, deterministic chaos). These uncertainties must be assessed and managed for credible computational predictions.

Over the years two different types of approaches have emerged to quantify uncertainty in mechanical systems. In the parametric approach the uncertainties associated with the system parameters, such as Young's modulus, mass density, Poisson's ratio, damping coefficient and geometric parameters are quantified using statistical methods and propagated, for example, using the stochastic finite element method..$^{2-12}$ Model uncertainty or computational uncertainty on the other hand do not always explicitly depend on the system parameters. For example, there can be unquantified errors associated with the equation of motion (linear on non-linear), in the damping model (viscous or non-viscous ${ }^{13}$ ), in the model of structural joints, and also in the numerical methods (e.g, discretisation of displacement fields, truncation and roundoff errors, tolerances in the optimization and iterative algorithms, step-sizes in the time-integration methods). The parametric approach is not quite suitable to quantify this type of uncertainties. As a result non-parametric approaches ${ }^{14-22}$ have been proposed for this purpose.

In spite of extensive research over the past four decades we still lack a general purpose probabilistic predictive code for structural dynamical systems. The main reasons behind this are: (a) the computational time can be prohibitively high compared to a deterministic analysis for real problems, (b) the volume of input data can be unrealistic to obtain for a credible probabilistic analysis, (c) the predictive accuracy can be poor if considerable resources are not spend on the previous two items, and (d) as the state-of-the art methodology stands now (such as the Stochastic Finite Element Method), only very few highly trained professionals (such as those with PhDs) can even attempt to apply the complex concepts (e.g., random fields) and methodologies to real-life problems. One needs to strike a balance between these four issues. This paper is aimed at developing a new approach with the ambition that it should:

(a) not take more than 10 times the computational time required for the corresponding deterministic approach;

(b) result a predictive accuracy within $10 \%$ of direct Monte Carlo Simulation (MCS);

2 of 15

American Institute of Aeronautics and Astronautics 
(c) use no more than 10 times of input data needed for the corresponding deterministic approach; and

(d) enable 'normal' engineering graduates to perform probabilistic structural dynamic analyses with a reasonable amount of training.

A Wishart random matrix based approach is developed in this paper. First we briefly outline some aspects of random matrix theory in Section II. Parameter selection of Wishart matrices is discussed in Section III. Section IV closed-form expressions for the mean and covariance of the dynamic response of a linear system is derived in the frequency domain. In addition to the deterministic analysis, only the normalized standard deviation of the mass and stiffness matrices is required in this approach. The closed-form expressions enable very fast calculation of the response statistics at the cost of loss of accuracy with respect to direct MCS. A numerical example is given in Section V to explore different trade-offs being made in the proposed approach.

\section{Background of the Random Matrix Model for Structural Dynamics}

\section{A. The Equation of Motion}

The equation of motion of a damped $n$-degree-of-freedom linear dynamic system can be expressed as

$$
\mathbf{M} \ddot{\mathbf{q}}(t)+\mathbf{C} \dot{\mathbf{q}}(t)+\mathbf{K q}(t)=\mathbf{f}(t)
$$

where $\mathbf{M}, \mathbf{C}$ and $\mathbf{K}$ are the mass, damping and stiffness matrices respectively. In the presence of uncertainty in the system and model, the system matrices become random matrices. The probability density functions of the random matrices $\mathbf{M}, \mathbf{C}$ and $\mathbf{K}$ completely quantify the uncertainties associated with system (1). Given the uncertainties in the system matrices, the aim of the 'forward problem' is to obtain uncertainty associated with the response vector q. So far only direct Monte Carlo simulation based methods have been proposed when $\mathbf{M}, \mathbf{C}$ and $\mathbf{K}$ are modelled by random matrices. In this paper we develop analytical methods to obtain the response statistics of linear dynamical systems. The proposed approach is based on the direct inversion of complex random matrices.

\section{B. Brief Overview of Random Matrices}

Random matrices were introduced by Wishart ${ }^{23}$ in the late 1920 s in the context of multivariate statistics. Since then research on random matrices has attracted interests in physics, number theory and more recently in mechanical and electrical engineering. We refer the books ${ }^{24-29}$ for the history and applications of random matrix theory. The probability density function of a random matrix can be defined in a manner similar to that of a random variable or random vector. If $\mathbf{A}$ is a $n \times m$ real random matrix, then the matrix variate probability density function of $\mathbf{A} \in \mathbb{R}^{n \times m}$, denoted by $p_{\mathbf{A}}(\mathbf{A})$, is a mapping from the space of $n \times m$ real matrices to the real line, i.e., $p_{\mathbf{A}}(\mathbf{A}): \mathbb{R}^{n \times m} \rightarrow \mathbb{R}$. Here we define probability density functions of two random matrices which are relevant to stochastic mechanics problems.

Gaussian random matrix: A rectangular random matrix $\mathbf{X} \in \mathbb{R}^{n \times p}$ is said to have a matrix variate Gaussian distribution with mean matrix $\mathbf{M} \in \mathbb{R}^{n \times p}$ and covariance matrix $\boldsymbol{\Sigma} \otimes \boldsymbol{\Psi}$, where $\boldsymbol{\Sigma} \in \mathbb{R}_{n}^{+}$and $\boldsymbol{\Psi} \in \mathbb{R}_{p}^{+}$provided the pdf of $\mathbf{X}$ is given by

$$
p_{\mathbf{X}}(\mathbf{X})=(2 \pi)^{-n p / 2} \operatorname{det}\{\boldsymbol{\Sigma}\}^{-p / 2} \operatorname{det}\{\boldsymbol{\Psi}\}^{-n / 2} \operatorname{etr}\left\{-\frac{1}{2} \boldsymbol{\Sigma}^{-1}(\mathbf{X}-\boldsymbol{\mathcal { M }}) \boldsymbol{\Psi}^{-1}(\mathbf{X}-\boldsymbol{\mathcal { M }})^{T}\right\}
$$

This distribution is usually denoted as $\mathbf{X} \sim N_{n, p}(\mathcal{M}, \boldsymbol{\Sigma} \otimes \mathbf{\Psi})$.

$$
3 \text { of } 15
$$


Wishart matrix: A $n \times n$ symmetric positive definite random matrix $\mathbf{S}$ is said to have a Wishart distribution with parameters $p \geq n$ and $\boldsymbol{\Sigma} \in \mathbb{R}_{n}^{+}$, if its pdf is given by

$$
p_{\mathbf{S}}(\mathbf{S})=\left\{2^{\frac{1}{2} n p} \Gamma_{n}\left(\frac{1}{2} p\right) \operatorname{det}\{\boldsymbol{\Sigma}\}^{\frac{1}{2} p}\right\}^{-1}|\mathbf{S}|^{\frac{1}{2}(p-n-1)} \operatorname{etr}\left\{-\frac{1}{2} \boldsymbol{\Sigma}^{-1} \mathbf{S}\right\}
$$

where $\operatorname{etr}\{\bullet\}$ is the exponential of trace of a matrix. This distribution is usually denoted as $\mathbf{S} \sim W_{n}(p, \boldsymbol{\Sigma})$. Previous works ${ }^{16,19,20}$ showed that the system matrices arising in linear structural dynamics can be modelled as Wishart matrices. Originally ${ }^{23}$ only integer values were considered for the shape parameter $p$ in the Wishart distribution. However, it is a misconception that $p$ is limited to integer numbers only (see for example pp. 87 in Muirhead ${ }^{28}$ and pp. 89 in Gupta and $\mathrm{Nagar}^{30}$ ). One can easily observe that equation (3) is valid for all real $p$ greater than $n$ (when $p<n$, then the distribution is often called the anti-Wishart distribution ${ }^{31}$ ). In this paper we use the Wishart distribution in the generalized sense to allow the parameter $p$ as a real number. Note that Wishart distribution with parameter $p$ as a real number is also known as the matrix variate Gamma distribution of type I. ${ }^{30}$

In Eq. (3) the function $\Gamma_{n}(a)$ is the multivariate gamma function, which can be expressed in terms of products of the usual univariate gamma functions as

$$
\Gamma_{n}(a)=\pi^{\frac{1}{4} n(n-1)} \prod_{k=1}^{n} \Gamma\left[a-\frac{1}{2}(k-1)\right] ; \quad \text { for } \quad \Re(a)>\frac{1}{2}(n-1) .
$$

For more details on the matrix variate distributions we refer to the books. ${ }^{25-28,30}$

\section{Wishart Random Matrix Model for the System Matrices}

Suppose that the mean values of $\mathbf{M}, \mathbf{C}$ and $\mathbf{K}$ are given by $\mathbf{M}_{0}, \mathbf{C}_{0}$ and $\mathbf{K}_{0}$ respectively. This information is likely to be available, for example, using the deterministic finite element method. However, there are uncertainties associated with our modelling so that $\mathbf{M}, \mathbf{C}$ and $\mathbf{K}$ are actually random matrices. The matrix variate distributions of the random system matrices should be such that: (1) $\mathbf{M}, \mathbf{C}$ and $\mathbf{K}$ are symmetric matrices, (2) $\mathbf{M}$ is positive-definite and $\mathbf{C}$ and $\mathbf{K}$ are nonnegative-definite matrices. Because the system matrices are symmetric and non-negative definite, they can be always factorized as $\mathbf{G}=\mathbf{X} \mathbf{X}^{T}$. Here $\mathbf{G}$ represent any one the system matrices and $\mathbf{X}$ is in general a rectangular matrix. In the simplest case when $\mathbf{X}$ is considered as a Gaussian random matrix $\mathbf{X} \sim N_{n, p}(\mathbf{O}, \mathbf{\Sigma} \otimes \mathbf{I})$, the distribution of $\mathbf{G}=\mathbf{X X}^{T}$ becomes Wishart distribution ${ }^{23}$ so that $\mathbf{G} \sim W_{n}(p, \boldsymbol{\Sigma})$. This distribution has two unknown parameters, namely the scalar $p$ and the $n \times n$ matrix $\boldsymbol{\Sigma}$ which need to be fitted from 'data'. In this case by 'data' we mean the mean and standard deviation of the system matrices. The mean and standard deviation of the system matrices can be obtained in various ways including, stochastic finite element discretisation, experimental identification or direct Monte Carlo Simulation.

The normalized standard deviation of a random matrix $\mathbf{G}$ is defined as

$$
\sigma_{G}^{2}=\frac{\mathrm{E}\left[\|\mathbf{G}-\mathrm{E}[\mathbf{G}]\|_{\mathrm{F}}^{2}\right]}{\|\mathrm{E}[\mathbf{G}]\|_{\mathrm{F}}^{2}} .
$$

Assuming that $\mathbf{G}_{0}$ and $\sigma_{G}$ are known, there are four different ways in which the two parameters of the Wishart distribution can be selected:

1. The mean of the distribution is same as the 'measured' mean and the (normalized) standard deviation is same as the measured standard deviation. Mathematically, this implies

$$
\mathrm{E}[\mathbf{G}]=\mathbf{G}_{0} \quad \text { and } \quad \sigma_{G}=\widetilde{\sigma}_{G}
$$

$$
4 \text { of } 15
$$


where $\widetilde{\sigma}_{G}$ indicates measured standard deviation of a system matrix. This approach was proposed by Soize ${ }^{16}$ and it results in the following parameters for the Wishart distribution:

$$
p=n+1+\theta \quad \text { and } \quad \boldsymbol{\Sigma}=\mathbf{G}_{0} / p
$$

where

$$
\theta=(1+\beta) / \widetilde{\sigma}_{G}^{2}-(n+1)
$$

with

$$
\beta=\left\{\operatorname{Trace}\left(\mathbf{G}_{0}\right)\right\}^{2} / \operatorname{Trace}\left(\mathbf{G}_{0}^{2}\right) .
$$

With this distribution the difference between the mean of the inverse and the inverse of the mean of $\mathbf{G}$ can be significantly different. ${ }^{19,20}$

2. The distribution of $\mathbf{G}$ is such that $\mathrm{E}[\mathbf{G}]$ and $\mathrm{E}\left[\mathbf{G}^{-1}\right]$ are closest to measured $\mathbf{G}_{0}$ and $\mathbf{G}_{0}{ }^{-1}$ together with the normalized standard deviation of $\mathbf{G}$ equals to measured $\widetilde{\sigma}_{G}$. Mathematically, this implies

$$
\left\|\mathbf{G}_{0}-\mathrm{E}[\mathbf{G}]\right\|_{\mathrm{F}} \text { and }\left\|\mathbf{G}_{0}^{-1}-\mathrm{E}\left[\mathbf{G}^{-1}\right]\right\|_{\mathrm{F}} \text { are minimum and } \sigma_{G}=\widetilde{\sigma}_{G} .
$$

This approach was proposed by Adhikari ${ }^{19,20}$ and it results in the following parameters for the Wishart distribution:

$$
p=n+1+\theta \quad \text { and } \quad \boldsymbol{\Sigma}=\mathbf{G}_{0} / \alpha
$$

where

$$
\alpha=\sqrt{\theta(n+1+\theta)}
$$

and $p$ and $\theta$ are as defined in the previous case.

3. The mean of the inverse of the distribution is same as the 'measured' inverse of the mean matrix and the (normalized) standard deviation is same as the measured standard deviation. Mathematically, this implies

$$
\mathrm{E}\left[\mathbf{G}^{-1}\right]=\mathbf{G}_{0}^{-1} \text { and } \sigma_{G}=\widetilde{\sigma}_{G} .
$$

This approach was proposed by Adhikari ${ }^{22}$ and it results in the following parameters for the Wishart distribution:

$$
p=n+1+\theta \quad \text { and } \quad \boldsymbol{\Sigma}=\mathbf{G}_{0} / \theta
$$

where $p$ and $\theta$ are as defined in the first case.

4. The mean of the eigenvalues of the distribution is same as the 'measured' eigenvalues of the mean matrix and the (normalized) standard deviation is same as the measured standard deviation. Mathematically, this implies

$$
\mathrm{E}\left[\mathbf{M}^{-1}\right]=\mathbf{M}_{0}^{-1}, \mathrm{E}[\mathbf{K}]=\mathbf{K}_{0}, \sigma_{M}=\widetilde{\sigma}_{M} \quad \text { and } \quad \sigma_{K}=\widetilde{\sigma}_{K} .
$$

This approach was discussed by Adhikari ${ }^{22}$ and the resulting parameters can be easily obtained by following cases 1 and 3 above.

Based on numerical studies conducted by Adhikari, ${ }^{22}$ is was observed that parameter selection corresponding to case 3 produces the best fidelity with direct Monte Carlo simulation, followed by cases 2, 1, and 4. As a result, the parameters corresponding to case 3 are considered in the next section.

$$
5 \text { of } 15
$$




\section{Response Variability of Linear Stochastic Systems}

Taking the Fourier transform Eq. (1), the dynamic response of the system can be expressed in the Frequency domain as

$$
\mathbf{q}(\omega)=\mathbf{D}^{-1}(\omega) \mathbf{f}(\omega)
$$

where the dynamic stiffness matrix is defined as

$$
\mathbf{D}(\omega)=-\omega^{2} \mathbf{M}+i \omega \mathbf{C}+\mathbf{K} .
$$

This is a complex symmetric random matrix. The calculation of the response statistics requires the calculation of statistical moments of the inverse of this matrix. At this point two approaches can be taken, namely (a) approach based on random eigenvalues and eigenvectors, ${ }^{8,32,33}$ or (b) approach based on direct matrix inversion. In this paper the second approach is adopted. In order to proceed we employ the following assumptions:

1. Damping matrix is 'small' compared to the mass and stiffness matrices. This 'light damping' assumption is often made for a wide range of aero-mechanical systems. Note that no assumption regarding proportional damping ${ }^{34,35}$ is made here.

2. Thee damping matrix is deterministic. This assumption is often known as the constant loss factor assumption normally employed in high and mid-frequency vibration analysis.

3. The mass and stiffness matrices are statistically independent Wishart matrices.

4. The input force is deterministic.

Note that no assumptions related to small randomness or Gaussianity, often employed in perturbation based analyses, is made here. From Eq. (17) observe that for a given frequency value the dynamic stiffness matrix is linear combination two Wishart matrices with a deterministic imaginary 'shift'. The dynamic stiffness matrix is however cannot be a complex Wishart matrix as it is not Hermitian. Considering the fact that engineering interest often lies in the absolute value of the response, we are interested in the statistical moments of

$$
|\mathbf{q}|(\omega)=\left|\mathbf{D}^{-1}(\omega)\right||\mathbf{f}(\omega)|=|\mathbf{D}(\omega)|^{-1}|\mathbf{f}(\omega)|
$$

where the absolute of the dynamic stiffness matrix is given by

$$
|\mathbf{D}(\omega)|=\left\{\left[-\omega^{2} \mathbf{M}+\mathbf{K}\right]^{2}+\omega^{2} \mathbf{C}^{2}\right\}^{1 / 2} .
$$

For notational convenience the functional dependence of $\omega$ will be omitted. The first-order moment of the absolute of the response $|\mathbf{q}|$ can be calculated as

$$
\overline{\mathbf{q}}=\mathrm{E}[|\mathbf{q}|]=\mathrm{E}\left[|\mathbf{D}|^{-1}\right] \overline{\mathbf{f}}
$$

where $\overline{\mathbf{f}}=|\mathbf{f}|$. The second-order moment of the absolute of the response can be calculated as

$$
\begin{aligned}
\operatorname{cov}_{|\mathbf{q}|} & =\mathrm{E}\left[(|\mathbf{q}|-\mathrm{E}[|\mathbf{q}|])(|\mathbf{q}|-\mathrm{E}[|\mathbf{q}|])^{T}\right]=\mathrm{E}\left[|\mathbf{q}||\mathbf{q}|^{T}\right]-\overline{\mathbf{q}} \overline{\mathbf{q}}^{T} \\
& =\mathrm{E}\left[|\mathbf{D}|^{-1} \overline{\mathbf{f}} \overline{\mathbf{f}}^{T}|\mathbf{D}|^{-1}\right]-\overline{\mathbf{q}} \overline{\mathbf{q}}^{T} .
\end{aligned}
$$

The dynamic response statistics is obtained in two steps:

- A Wishart distribution is fitted to $|\mathbf{D}|$ matrix, which is symmetric and non-negative definite. Note that $\mathbf{D}$ cannot be a Wishart matrix unless the system is undamped.

- Once the parameters of the Wishart distribution corresponding to $|\mathbf{D}|$ is identified, the inverse moments are obtained exactly in closed-from using the inverted Wishart distribution. ${ }^{30}$

$$
6 \text { of } 15
$$




\section{A. Random Dynamic Stiffness Matrix}

We consider that $|\mathbf{D}|(\omega) \sim W_{n}\left(p_{D}, \boldsymbol{\Sigma}_{D}\right)=\mathbf{S}$ where $p_{D}(\omega) \in \mathbb{R}$ and $\boldsymbol{\Sigma}_{D}(\omega) \in \mathbb{R}^{n \times n}$ are the unknown parameters of the Wishart distribution to be identified. Clearly various approaches can be used to fit these parameters. Here we employ the following criteria

- The square-root of the mean of $|\mathbf{D}|^{2}$ is same as the mean of $\mathbf{S}$, that is

$$
\sqrt{\mathrm{E}\left[|\mathbf{D}|^{2}\right]}=\mathrm{E}[\mathbf{S}]
$$

- The standard deviation of of $|\mathbf{D}|^{2}$ is same as the standard deviation of $\mathbf{S}^{2}$, that is

$$
\sigma_{|\mathbf{D}|^{2}}=\sigma_{\mathbf{S}^{2}}
$$

For the first criteria we have

$$
p_{D} \boldsymbol{\Sigma}_{D}=\left\{\left(-\omega^{2} p_{M} \boldsymbol{\Sigma}_{M}+p_{K} \boldsymbol{\Sigma}_{K}\right)^{2}+\omega^{2} \mathbf{C}^{2}\right\}^{1 / 2} .
$$

For the second criteria we have

$$
\omega^{4} \sigma_{M}^{2}+\sigma_{K}^{2}=\sigma_{D}^{2}
$$

because the damping matrix is assumed to be deterministic. Using the expression of the second moment of the Wishart matrices ${ }^{30}$ the preceding equation can be expressed as

$\omega^{4} p_{M}\left(\mathbf{M}_{0}^{2}+\mathbf{M}_{0} \operatorname{Trace}\left(\mathbf{M}_{0}\right)\right) / \theta_{M}+p_{K}\left(\mathbf{K}_{0}^{2}+\mathbf{K}_{0} \operatorname{Trace}\left(\mathbf{K}_{0}\right)\right) / \theta_{K}=\left(|\mathbf{D}|^{2}+|\mathbf{D}| \operatorname{Trace}(|\mathbf{D}|)\right) / p_{D}$.

The scalar $p_{D}(\omega)$ can be obtained by the least-square error minimization as

$$
p_{D}(\omega)=\operatorname{Trace}(\mathcal{A B}) / \operatorname{Trace}\left(\mathcal{A}^{2}\right)
$$

where

$$
\begin{aligned}
\mathcal{A} & =\omega^{4} p_{M}\left(\mathbf{M}_{0}{ }^{2}+\mathbf{M}_{0} \operatorname{Trace}\left(\mathbf{M}_{0}\right)\right) / \theta_{M}+p_{K}\left(\mathbf{K}_{0}{ }^{2}+\mathbf{K}_{0} \operatorname{Trace}\left(\mathbf{K}_{0}\right)\right) / \theta_{K} \\
\text { and } \mathcal{B} & =|\mathbf{D}(\omega)|^{2}+|\mathbf{D}(\omega)| \operatorname{Trace}(|\mathbf{D}(\omega)|) .
\end{aligned}
$$

From Eq. (24), the matrix $\boldsymbol{\Sigma}_{D}$ can be obtained as

$$
\boldsymbol{\Sigma}_{D}(\omega)=|\mathbf{D}|(\omega) / p_{D}(\omega) .
$$

Equations (27) and (30) completely define the parameters of the Wishart distribution corresponding to $|\mathbf{D}|(\omega)$ for each $\omega$. Next we utilize this distribution to obtain the response statistics in an exact manner.

\section{B. Second-order Statistics of Dynamic Response}

If $\mathbf{S}$ is a Wishart matrix, then the distribution of $\mathbf{V}=\mathbf{S}^{-1}$ is known as the inverted Wishart distribution $^{28,30}$ and is defined as below:

Inverted Wishart matrix: A $n \times n$ symmetric positive definite random matrix $\mathbf{V}$ is said to have an inverted Wishart distribution with parameters $m$ and $\boldsymbol{\Psi} \in \mathbb{R}_{n}^{+}$, if its pdf is given by

$$
p_{\mathbf{V}}(\mathbf{V})=\frac{2^{-\frac{1}{2}(m-n-1) n \operatorname{det}\{\boldsymbol{\Psi}\}^{\frac{1}{2}(m-n-1)}}}{\Gamma_{n}\left(\frac{1}{2}(m-n-1)\right) \operatorname{det}\{\mathbf{V}\}^{m / 2}} \operatorname{etr}\left\{-\frac{1}{2} \mathbf{V}^{-1} \mathbf{\Psi}\right\} ; \quad m>2 n, \mathbf{\Psi}>0
$$

$$
7 \text { of } 15
$$


This distribution is usually denoted as $\mathbf{V} \sim I W_{n}(m, \mathbf{\Psi})$.

Moments of the inverted Wishart matrix can be obtained exactly in closed-form. Here we utilize these powerful results to obtain the second-order moments of the inverse of the dynamic stiffness matrix. Following Gupta and $\operatorname{Nagar}^{30}$ (pp. 99, Theorem 3.3.16) we have

$$
\begin{aligned}
\mathrm{E}\left[\mathbf{S}^{-1}\right] & =\frac{1}{p_{D}-n-1} \boldsymbol{\Sigma}_{D}^{-1}=\frac{p_{D}(\omega)|\mathbf{D}|^{-1}(\omega)}{p_{D}(\omega)-n-1} \\
\mathrm{E}\left[\mathbf{S}^{-1} \mathbf{A} \mathbf{S}^{-1}\right] & =\frac{\operatorname{Trace}\left(\boldsymbol{\Sigma}_{D}^{-1} \mathbf{A}\right) \boldsymbol{\Sigma}_{D}^{-1}+\left(p_{D}-n-1\right) \boldsymbol{\Sigma}_{D}^{-1} \mathbf{A} \boldsymbol{\Sigma}_{D}^{-1}}{\left(p_{D}-n\right)\left(p_{D}-n-1\right)\left(p_{D}-n-3\right)} .
\end{aligned}
$$

Equation (21) can now be evaluated by considering $\mathbf{A}=\overline{\mathbf{f}} \overline{\mathbf{f}}^{T}$. Thus we have

$$
\begin{aligned}
\operatorname{cov}_{|\mathbf{q}|} & =\mathrm{E}\left[|\mathbf{D}|^{-1} \overline{\mathbf{f}} \overline{\mathbf{f}}^{T}|\mathbf{D}|^{-1}\right]-\overline{\mathbf{q}} \overline{\mathbf{q}}^{T} \\
& =\frac{\operatorname{Trace}\left(\boldsymbol{\Sigma}_{D}^{-1} \overline{\mathbf{f}} \overline{\mathbf{f}}^{T}\right) \boldsymbol{\Sigma}_{D}^{-1}+\left(p_{D}-n-1\right) \boldsymbol{\Sigma}_{D}^{-1} \overline{\mathbf{f}} \overline{\mathbf{f}}^{T} \boldsymbol{\Sigma}_{D}^{-1}}{\left(p_{D}-n\right)\left(p_{D}-n-1\right)\left(p_{D}-n-3\right)}-\overline{\mathbf{q}} \overline{\mathbf{q}}^{T} \\
& =\frac{\operatorname{Trace}\left(\overline{\mathbf{q}} \overline{\mathbf{f}}^{T}\right) \boldsymbol{\Sigma}_{D}^{-1}+\left(p_{D}-n-1\right) \overline{\mathbf{q}} \overline{\mathbf{q}}^{T}}{\left(p_{D}-n\right)\left(p_{D}-n-1\right)\left(p_{D}-n-3\right)}-\overline{\mathbf{q}} \overline{\mathbf{q}}^{T} .
\end{aligned}
$$

Further simplifying we have

$$
\overline{\mathbf{q}}=\frac{p_{D}(\omega)}{\theta_{D}(\omega)} \mathbf{q}_{0}(\omega)
$$

Here $\mathbf{q}_{0}(\omega)$ is the absolute value of the response corresponding to the baseline or 'mean' system

$$
\mathbf{q}_{0}(\omega)=\left|\mathbf{D}_{0}(\omega)\right|^{-1}|\mathbf{f}(\omega)|
$$

with

$$
\left|\mathbf{D}_{0}(\omega)\right|=\left|-\omega^{2} \mathbf{M}_{0}+i \omega \mathbf{C}+\mathbf{K}_{0}\right|
$$

and

$$
\theta_{D}(\omega)=p_{D}(\omega)-n-1 .
$$

After some simplification, the covariance of the absolute of the response can be obtained from Eq. (21) as

$$
\operatorname{cov}_{|\mathbf{q}|}(\omega)=\frac{\left(\theta_{D}(\omega)+n+1\right) \operatorname{Trace}\left(\mathbf{q}_{0}(\omega) \overline{\mathbf{f}}(\omega)^{T}\right) \boldsymbol{\Sigma}_{D}^{-1}(\omega)+\left(\theta_{D}(\omega)+2\right) \mathbf{q}_{0}(\omega) \mathbf{q}_{0}^{T}(\omega)}{\left(\theta_{D}(\omega)+1\right)\left(\theta_{D}(\omega)-2\right)}
$$

Equations (35) and (39) completely define the first two moments of the absolute value of the response of a general uncertain linear dynamical system in closed-form.

\section{Numerical Example: Dynamic Response of a Plate With Random Properties}

A cantilever steel plate with uncertain properties is considered to illustrate the application of the closed-form expressions based on Wishart random matrices in probabilistic structural dynamics. The diagram of the plate together with the deterministic numerical values assumed for the system parameters are shown in figure 1. The plate is excited by an unit harmonic force and the response is calculated at the point shown in the diagram. The standard four-noded thin plate bending element (resulting 12 degrees of freedom per element) is used. The plate is divided into 25 elements along

$$
8 \text { of } 15
$$




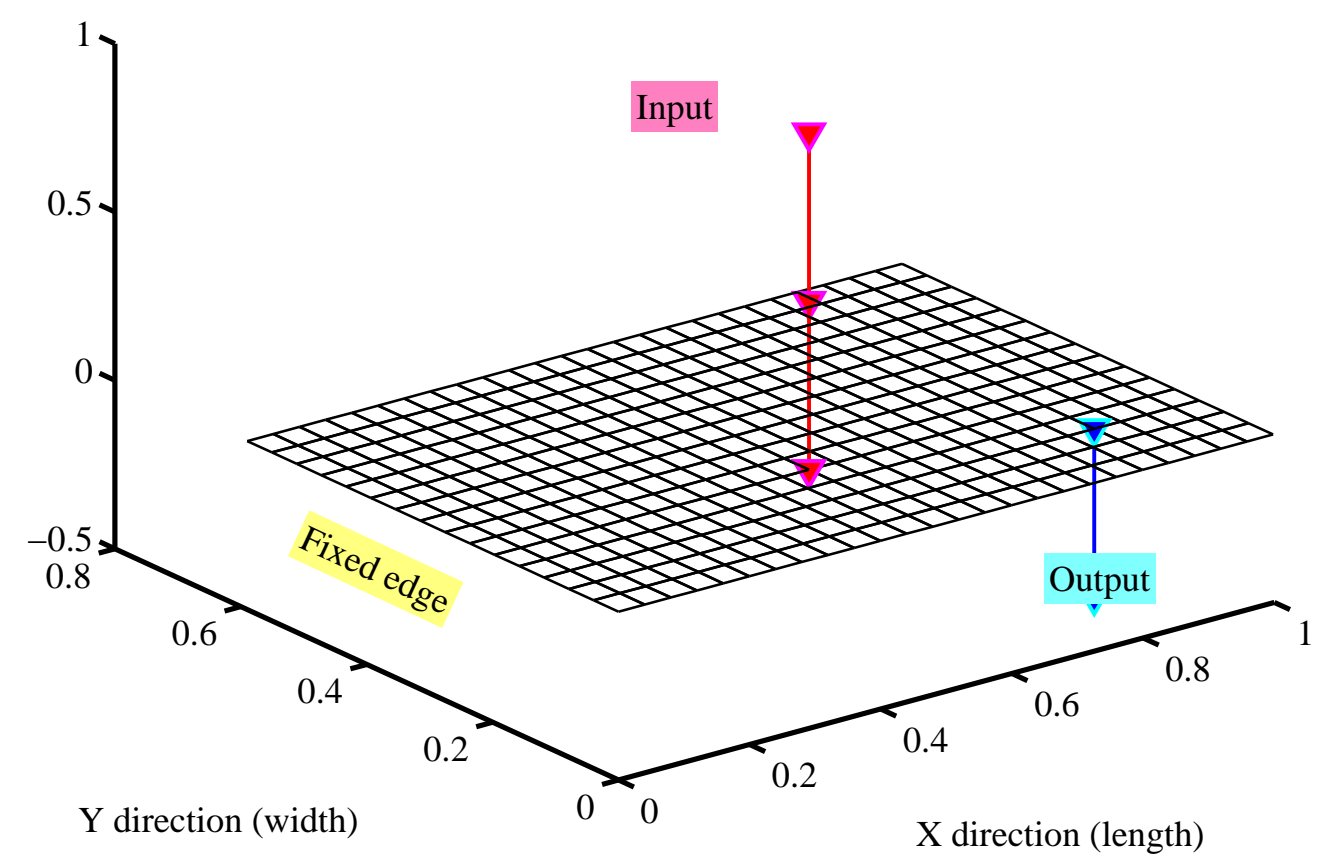

Figure 1. The Finite Element (FE) model of a steel cantilever plate: $25 \times 15$ elements, 416 nodes, 1200 degrees-of-freedom. The deterministic properties are: $\bar{E}=200 \times 10^{9} \mathbf{N} / \mathbf{m}^{2}, \bar{\mu}=0.3, \bar{\rho}=7860 \mathrm{~kg} / \mathbf{m}^{3}$, $\bar{t}=3.0 \mathrm{~mm}, L_{x}=0.998 \mathrm{~m}, L_{y}=0.59 \mathrm{~m}$. Input node number: 817 , output node number: 1000, modal damping factor: $0.5 \%$ for all modes.

the $\mathrm{x}$-axis and 15 elements along the $\mathrm{y}$-axis for numerical calculations. The resulting system has 1200 degrees of freedom so that $n=1200$. It is assumed that the Young's modulus, Poissons ratio, mass density and thickness are random fields of the form

$$
\begin{aligned}
E(\mathbf{x}) & =\bar{E}\left(1+\epsilon_{E} f_{1}(\mathbf{x})\right) \\
\mu(\mathbf{x}) & =\bar{\mu}\left(1+\epsilon_{\mu} f_{2}(\mathbf{x})\right) \\
\rho(\mathbf{x}) & =\bar{\rho}\left(1+\epsilon_{\rho} f_{3}(\mathbf{x})\right) \\
\text { and } \quad t(\mathbf{x}) & =\bar{t}\left(1+\epsilon_{t} f_{4}(\mathbf{x})\right) .
\end{aligned}
$$

The two dimensional vector $\mathbf{x}$ denotes the spatial coordinates. The strength parameters are assumed to be $\epsilon_{E}=0.15, \epsilon_{\mu}=0.10, \epsilon_{\rho}=0.14$ and $\epsilon_{t}=0.12$. The random fields $f_{i}(\mathbf{x}), i=1, \cdots, 4$ are assumed to be delta-correlated homogenous Gaussian random fields. Monte Carlo simulation is performed to obtain the FRFs.

We present results for a typical cross-FRF and a driving point FRF of the system. The following three approaches are compared to gain understanding into the proposed approximate method:

- Direct Monte Carlo Simulation: Here the response statistics is obtained using 1000 samples of the actual random fields describing the system. This is considered as the most exact description of the response statistics and used as the benchmark to evaluate the other approaches.

- Monte Carlo Simulation using Wishart Matrices: Here the response statistics is obtained using 1000 samples of the fitted Wishart matrices describing the mass and stuffiness matrices. The details of the parametric variations of the random fields are not used in the Wishart

$$
9 \text { of } 15
$$


matrix approach. From the simulated random mass and stiffness matrices we obtain $n=1200$, $\sigma_{M}=0.0999$ and $\sigma_{K}=0.2151$. Since $0.5 \%$ constant modal damping factor is assumed for all the modes, $\sigma_{C}=0$. The only uncertainty related information used in the random matrix approach are the values of $\sigma_{M}$ and $\sigma_{K}$. The information regarding which element property functions are random fields, nature of these random fields (correlation structure, Gaussian or non-Gaussian) and the amount of randomness are not used in the Wishart matrix approach. This is aimed to depict a realistic situation when the detailed information regarding uncertainties in a complex engineering system is not available to the analyst. Using $n=1200$, $\sigma_{M}=0.0999$ and $\sigma_{K}=0.2151$, together with the deterministic values of $\mathbf{M}$ and $\mathbf{K}$ we obtain $\theta_{M}=32950$ and $\theta_{K}=5779$. Using these, the samples of the mass and stiffness matrices are simulated following the procedure outlined in reference ${ }^{20}$ and the response statistics are calculated using standard statistical methods.

- Proposed Analytical Method using Wishart Matrices: Here the closed-form expressions given by Eqs. (35) and (39) are used to obtain the mean and standard-deviation of the response.

The mean of the amplitude of the response of the cross-FRF of the plate is shown in figure 2 . Percentage error in employing the Wishart Random Matrix approximation using Monte Carlo Simulation and the proposed analytical approach is also shown in the figure. The error using the analytical approach is similar to that obtained using the Monte Carlo Simulation of the Wishart matrices. Except for the very low frequency ranges, error resulted from the proposed analytical methods is generally less than $10 \%$. The mean of the amplitude of the response of the drivingpoint-FRF of the plate is shown in figure 3. Percentage error in employing the Wishart Random Matrix approximation using Monte Carlo Simulation and the proposed analytical approach is also shown in the figure. Like the previous case, error using the analytical approach is generally less than $10 \%$.

Now we turn our attention to the standard deviation of the amplitude of the FRFs. The standard deviation of the amplitude of the response of the cross-FRF of the plate is shown in figure 4. Percentage error in employing the Wishart Random Matrix approximation using Monte Carlo Simulation and the proposed analytical approach is also shown in the figure. The error using the analytical approach is of the same order to that obtained using the Monte Carlo Simulation of the Wishart matrices. Except for the very low frequency ranges, error resulted from the proposed analytical methods is generally less than $10 \%$. The standard deviation of the amplitude of the response of the driving-point-FRF of the plate is shown in figure 5. Percentage error in employing the Wishart Random Matrix approximation using Monte Carlo Simulation and the proposed analytical approach is also shown in the figure. Like the previous three figures, error using the analytical approach is generally less than $10 \%$.

The numerical results obtained here show that the proposed analytical method based on Wishart random matrices results in acceptable accuracy. Uncertainty related information needed for the proposed approach are the normalized standard deviations of the mass and stiffness matrices. The mean and covariance of the amplitude of the dynamic response in the frequency domain is obtained by simple post-processing of the results corresponding to the baseline system. This fact makes this approach particularly suitable to integrate with commercial finite element software.

\section{Conclusions}

The probabilistic characterization of the response of linear stochastic dynamical systems is considered. This problem requires the solution of a set of coupled complex algebraic equations, which in turn involves the inverse of a complex symmetric random matrix. Wishart random matrix model is used for the mass and stiffness matrices. In addition to the baseline matrices, only

10 of 15

American Institute of Aeronautics and Astronautics 


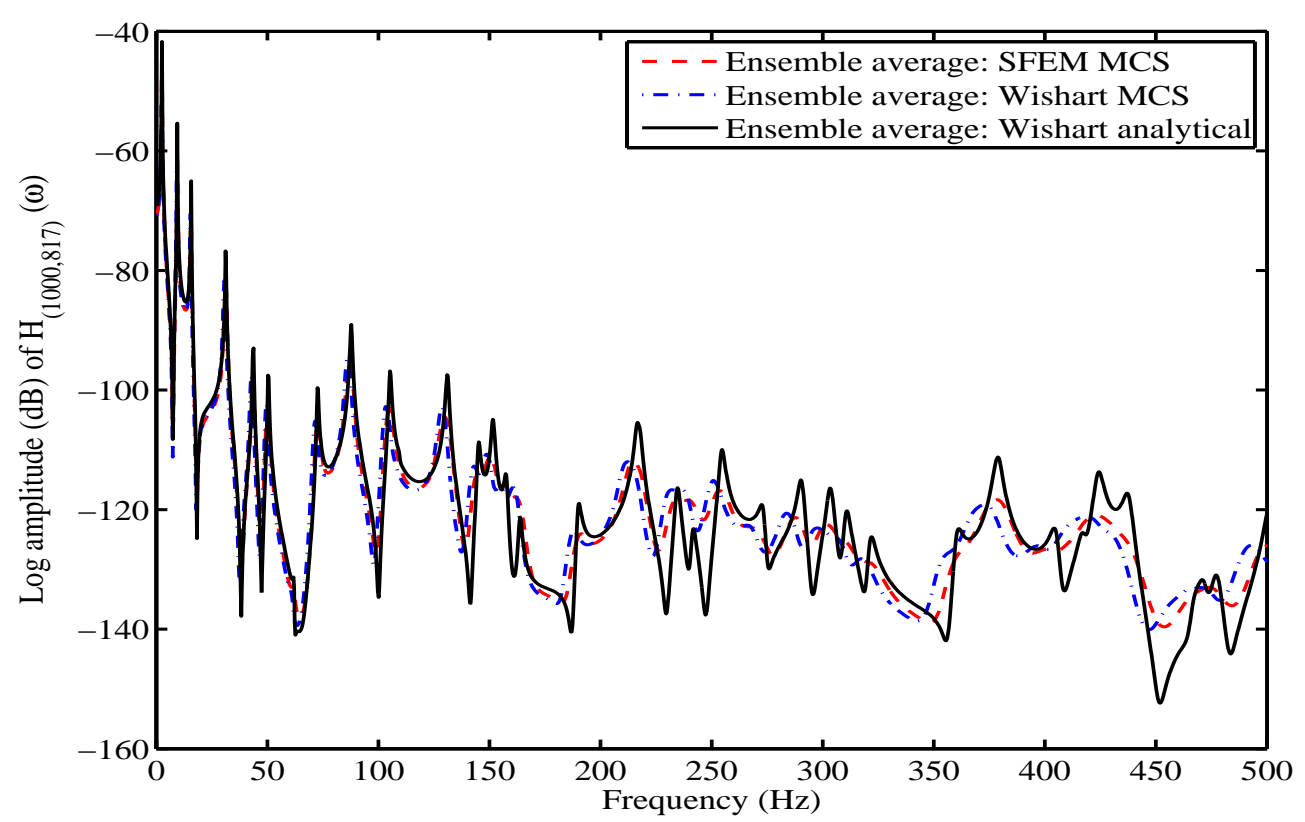

(a) Mean of the amplitude

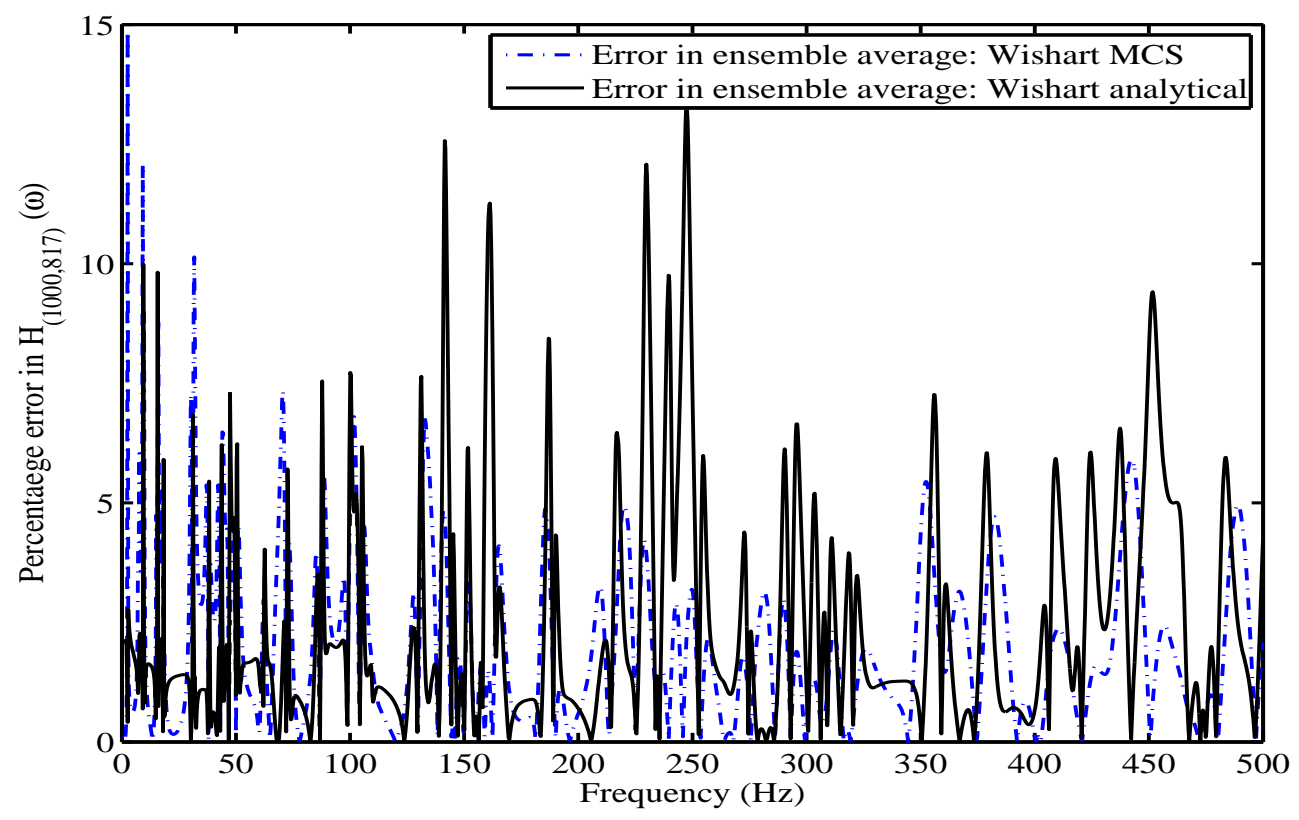

(b) Percentage error in employing the Wishart Random Matrix approximation

Figure 2. Mean of the amplitude of the response of the cross-FRF of the plate, $n=1200, \sigma_{M}=0.0999$ and $\sigma_{K}=0.2151$.

normalized standard deviations are required to obtain the parameters of the Wishart distributions. Assuming the damping is small and not random, a Wishart distribution is fitted to the amplitude of the dynamic stiffness matrix for every frequency point. Properties of the inverted Wishart distribution are used to obtain the statistics of the amplitude of the dynamic response. Approximate closed-form expressions of the mean and covariance of the amplitude of the dynamic response in the frequency domain is derived. These expressions are simple post-processing of the results corresponding to the baseline system. The method is applied to frequency response analysis of a 


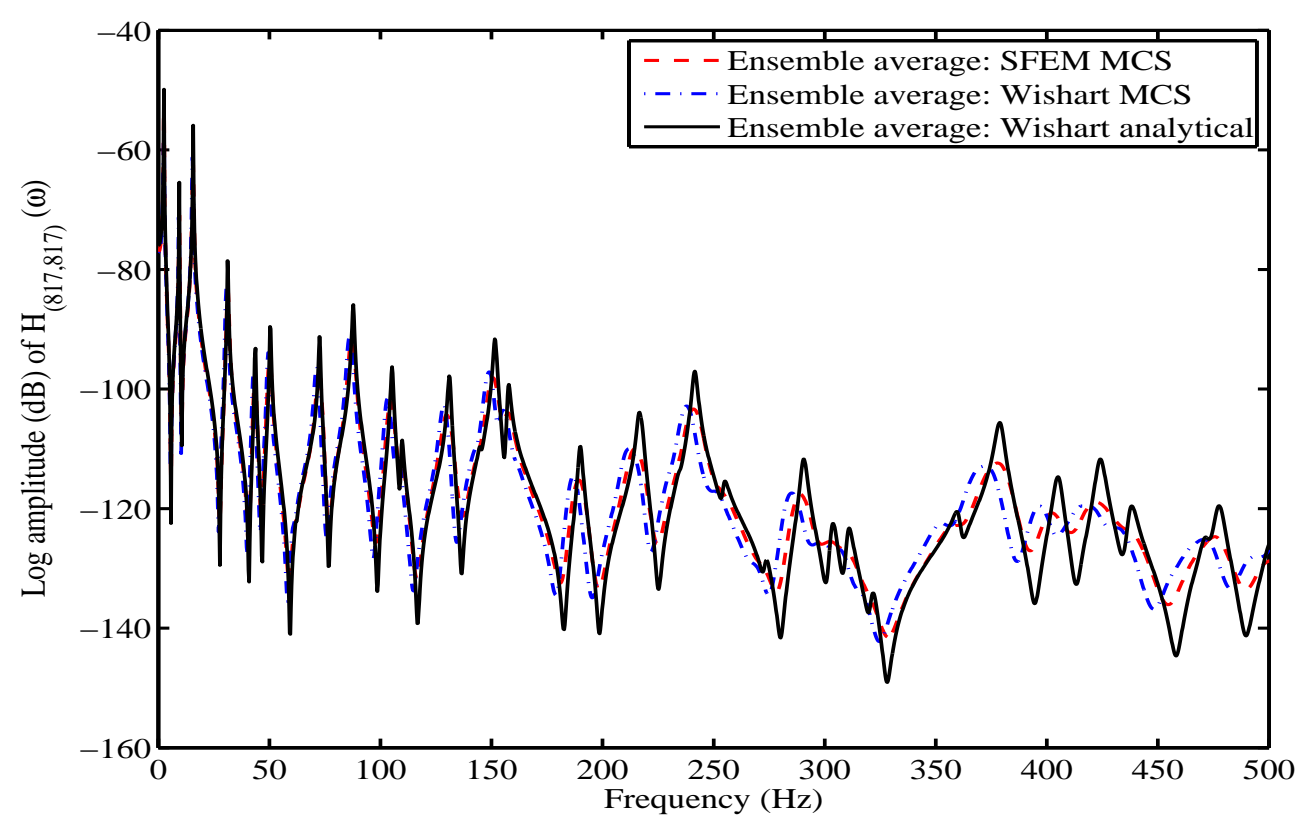

(a) Mean of the amplitude

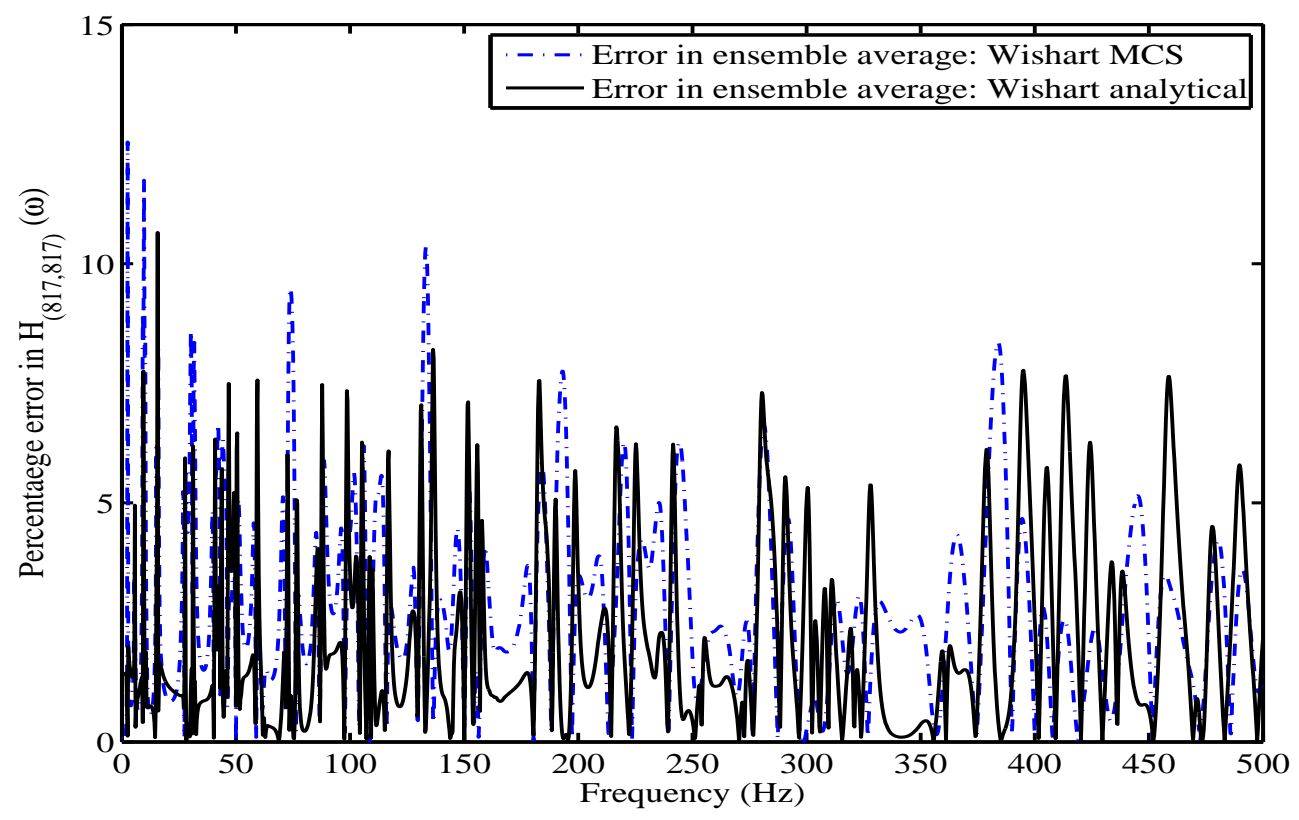

(b) Percentage error in employing the Wishart Random Matrix Approximation

Figure 3. Mean of the amplitude of the response of the driving-point-FRF of the plate, $n=1200$, $\sigma_{M}=0.0999$ and $\sigma_{K}=0.2151$.

cantilever plate with uncertainties. Error obtained using the proposed analytical method is less than $10 \%$ when compared with the results obtained from direct Monte Carlo simulation.

\section{Acknowledgments}

The author acknowledges the support of the UK Engineering and Physical Sciences Research Council (EPSRC) through the award of an Advanced Research Fellowship. 


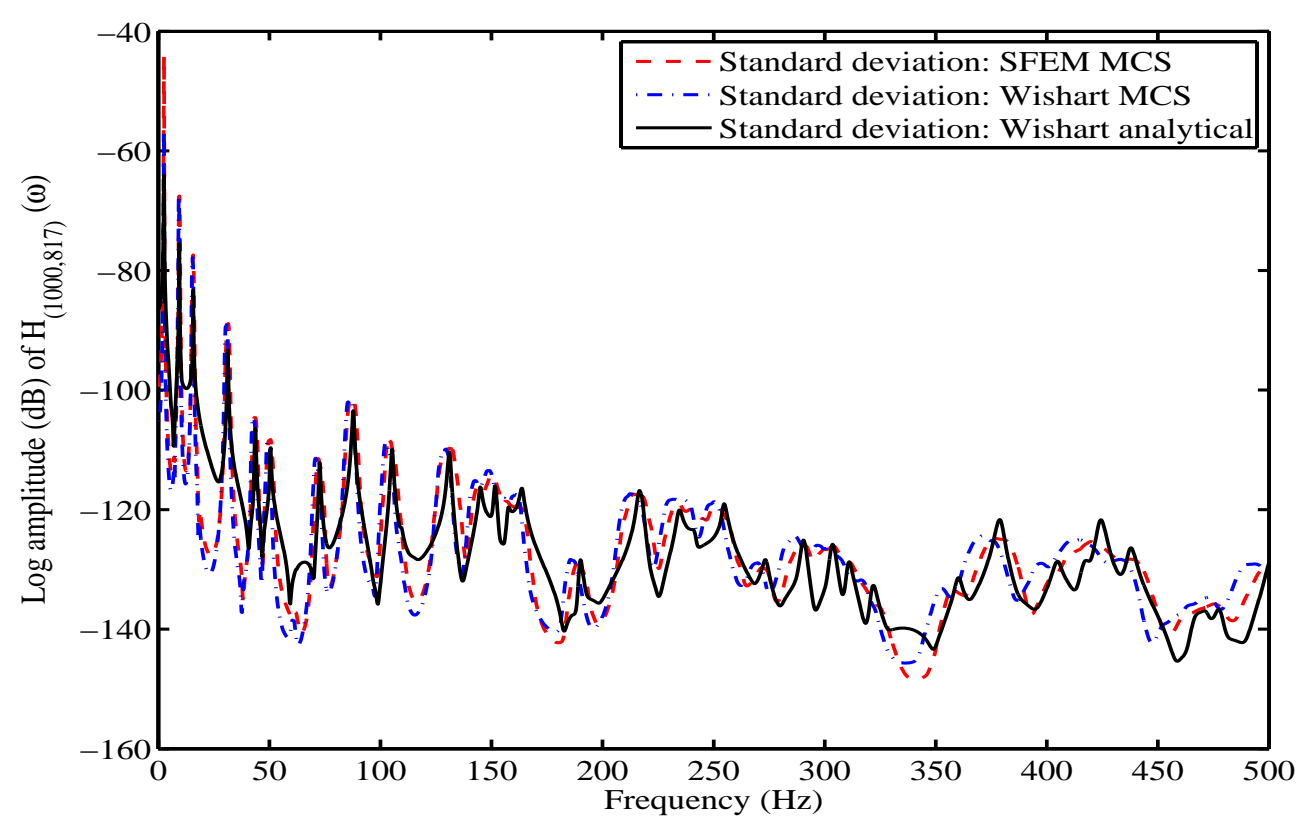

(a) Standard deviation of the amplitude

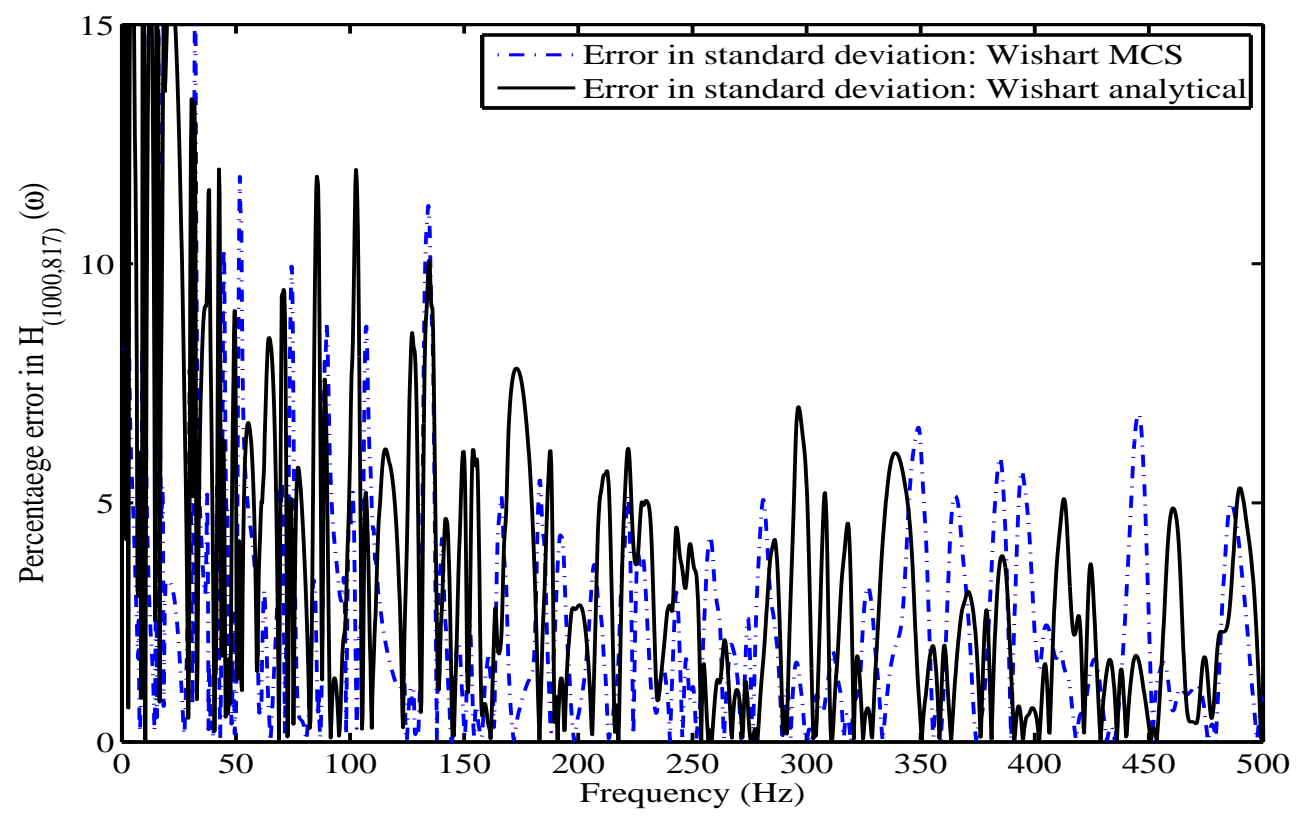

(b) Percentage error in employing the Wishart Random Matrix Approximation

Figure 4. Standard deviation of the amplitude of the response of the cross-FRF of the plate, $n=1200$, $\sigma_{M}=0.0999$ and $\sigma_{K}=0.2151$.

\section{References}

${ }^{1}$ Graham, A., Kronecker Products and Matrix Calculus With Applications, Mathematics and its Applications, Ellis Horwood Limited, Chichester, UK, 1981.

${ }^{2}$ Shinozuka, M. and Yamazaki, F., "Stochastic finite element analysis: an introduction," Stochastic structural dynamics: Progress in theory and applications, edited by S. T. Ariaratnam, G. I. Schuëller, and I. Elishakoff, Elsevier Applied Science, London, 1998.

${ }^{3}$ Ghanem, R. and Spanos, P., Stochastic Finite Elements: A Spectral Approach, Springer-Verlag, New York, USA, 1991. 


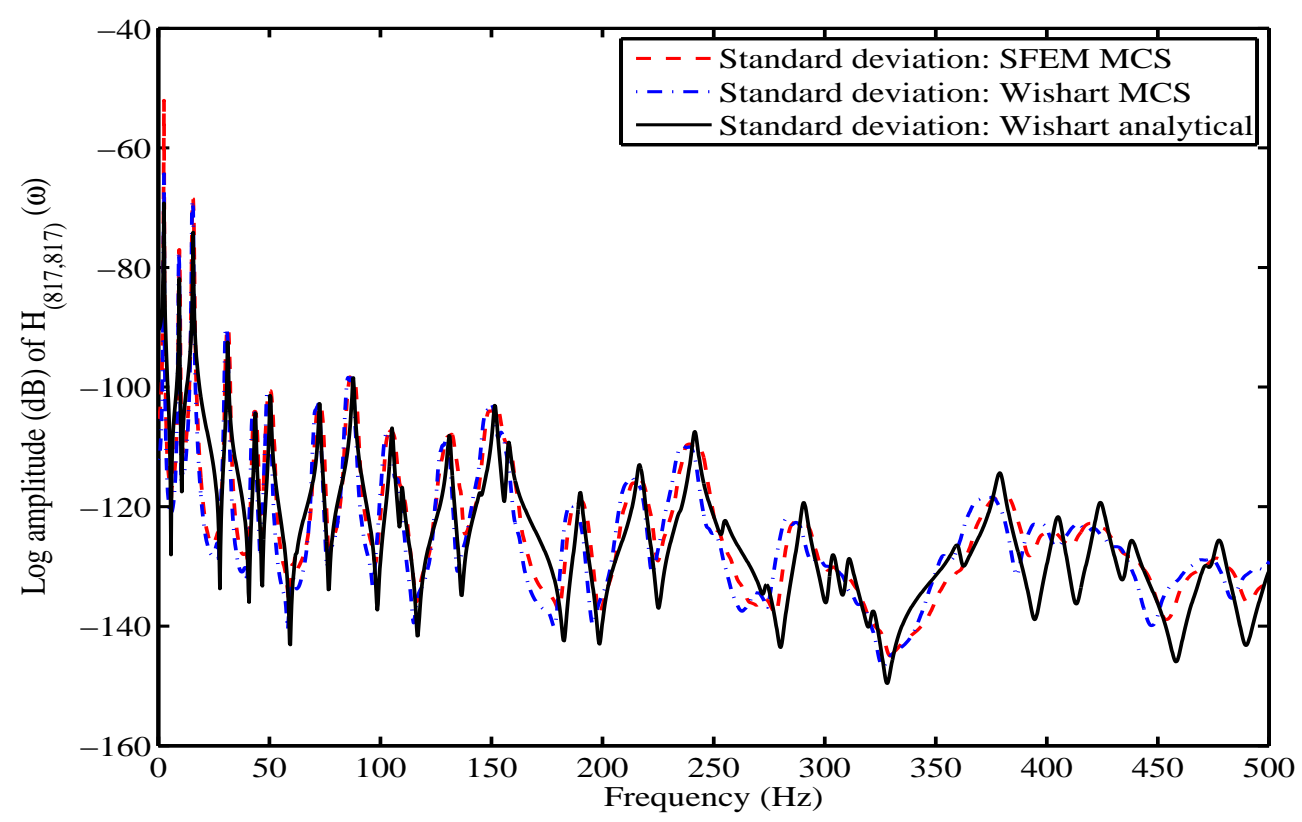

(a) Standard deviation of the amplitude

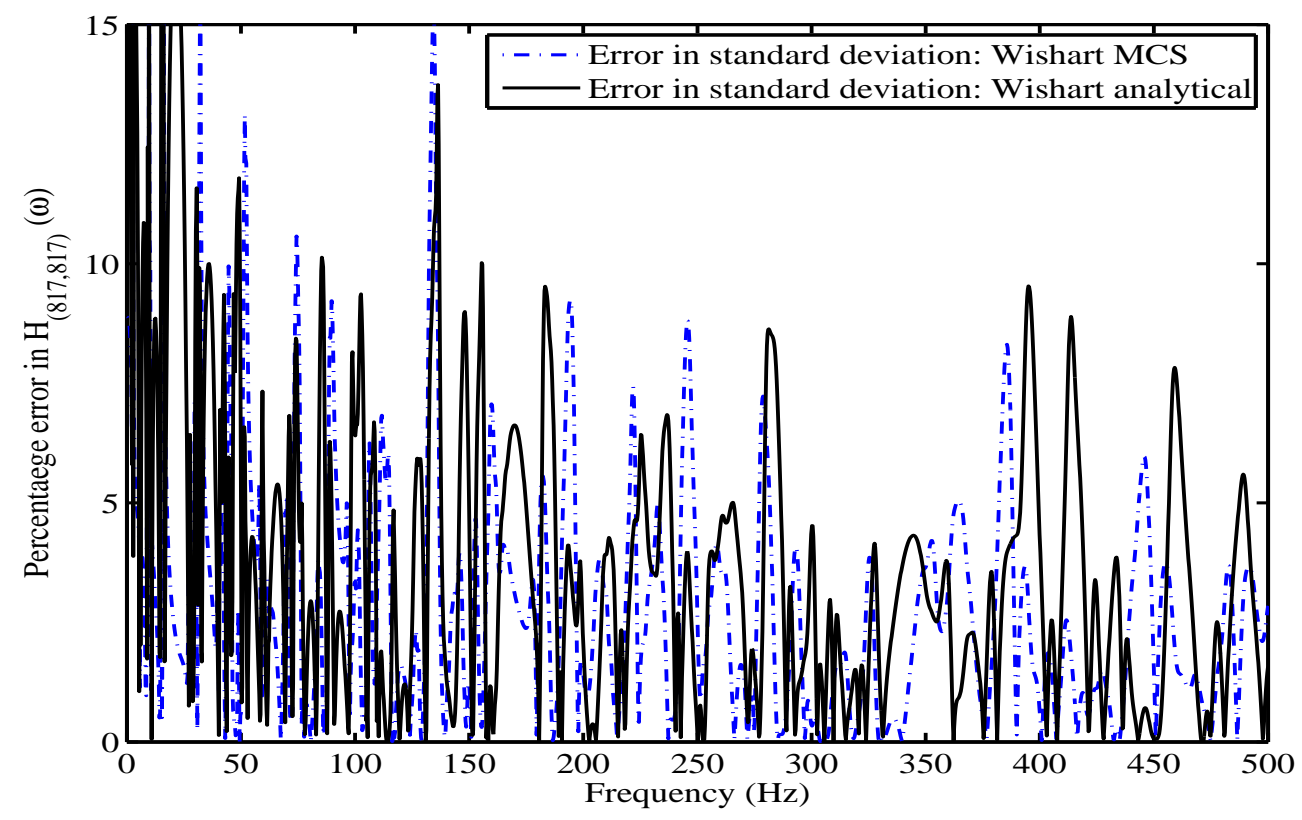

(b) Percentage error in employing the Wishart Random Matrix Approximation

Figure 5. Standard deviation of the amplitude of the response of the driving-point-FRF of the plate, $n=1200, \sigma_{M}=0.0999$ and $\sigma_{K}=0.2151$.

${ }^{4}$ Kleiber, M. and Hien, T. D., The Stochastic Finite Element Method, John Wiley, Chichester, 1992.

${ }^{5}$ Matthies, H. G., Brenner, C. E., Bucher, C. G., and Soares, C. G., "Uncertainties in probabilistic numerical analysis of structures and solids - Stochastic finite elements," Structural Safety, Vol. 19, No. 3, 1997, pp. $283-336$.

${ }^{6}$ Manohar, C. S. and Adhikari, S., "Dynamic stiffness of randomly parametered beams," Probabilistic Engineering Mechanics, Vol. 13, No. 1, January 1998, pp. 39-51.

${ }^{7}$ Manohar, C. S. and Adhikari, S., "Statistical analysis of vibration energy flow in randomly parametered trusses," Journal of Sound and Vibration, Vol. 217, No. 1, 1998, pp. 43-74.

${ }^{8}$ Adhikari, S. and Manohar, C. S., "Dynamic analysis of framed structures with statistical uncertainties," International Journal for Numerical Methods in Engineering, Vol. 44, No. 8, 1999, pp. 1157-1178.

\section{4 of 15}


${ }^{9}$ Adhikari, S. and Manohar, C. S., "Transient dynamics of stochastically parametered beams," ASCE Journal of Engineering Mechanics, Vol. 126, No. 11, November 2000, pp. 1131-1140.

${ }^{10}$ Haldar, A. and Mahadevan, S., Reliability Assessment Using Stochastic Finite Element Analysis, John Wiley and Sons, New York, USA, 2000.

${ }^{11}$ Sudret, B. and Der-Kiureghian, A., "Stochastic Finite Element Methods and Reliability," Tech. Rep. UCB/SEMM-2000/08, Department of Civil \& Environmental Engineering, University Of California, Berkeley, November 2000 .

${ }^{12}$ Elishakoff, I. and Ren, Y. J., Large Variation Finite Element Method for Stochastic Problems, Oxford University Press, Oxford, U.K., 2003.

${ }^{13}$ Adhikari, S., "On the quantification of damping model uncertainty," Journal of Sound and Vibration, Vol. 305, No. 1-2, September 2007, pp. 153-171.

${ }^{14}$ Langley, R. S., "A general derivation of the statistical energy analysis equations for coupled dynamic-systems," Journal of Sound and Vibration, Vol. 135, No. 3, December 1989, pp. 499-508.

${ }^{15}$ Langley, R. S. and Bremner, P., "A hybrid method for the vibration analysis of complex structural-acoustic systems," Journal of the Acoustical Society of America, Vol. 105, No. 3, March 1999, pp. 1657-1671.

${ }^{16}$ Soize, C., "A nonparametric model of random uncertainties for reduced matrix models in structural dynamics," Probabilistic Engineering Mechanics, Vol. 15, No. 3, 2000, pp. 277-294.

${ }^{17}$ Sarkar, A. and Ghanem, R., "A substructure approach for the midfrequency vibration of stochastic systems," Journal of the Acoustical Society of America, Vol. 113, No. 4, 2003, pp. 1922-1934, Part 1.

${ }^{18}$ Sarkar, A. and Ghanem, R., "Reduced models for the medium-frequency dynamics of stochastic systems," Journal of the Acoustical Society of America, Vol. 113, No. 2, 2003, pp. 834-846.

${ }^{19}$ Adhikari, S., "A non-parametric approach for uncertainty quantification in elastodynamics," 47 th AIAA/ASME/ASCE/AHS/ASC Structures, Structural Dynamics \& Materials Conference, Newport, Rhode Island, USA, May 2006.

${ }^{20}$ Adhikari, S., "Matrix variate distributions for probabilistic structural mechanics," AIAA Journal, Vol. 45, No. 7, July 2007, pp. 1748-1762.

${ }^{21}$ Adhikari, S., "An unified parametric-nonparametric uncertainty quantification approach for linear dynamical systems," 48th AIAA/ASME/ASCE/AHS/ASC Structures, Structural Dynamics \& Materials Conference, AIAA, Waikiki, Hawaii, USA, April 2007.

${ }^{22}$ Adhikari, S., "Wishart random matrices in probabilistic structural mechanics," ASCE Journal of Engineering Mechanics, 2008, accepted for publication.

${ }^{23}$ Wishart, J., "The generalized product moment distribution in samples from a normal multivariate population," Biometrika, Vol. 20, No. A, 1928, pp. 32-52.

${ }^{24}$ Mezzadri, F. and Snaith, N. C., editors, Recent Perspectives in Random Matrix Theory and Number Theory, London Mathematical Society Lecture Note, Cambridge, U. K., 2005, Cambridge University Press.

${ }^{25}$ Tulino, A. M. and Verdú, S., Random Matrix Theory and Wireless Communications, now Publishers Inc., Hanover, MA, USA, 2004.

${ }^{26}$ Eaton, M. L., Multivariate Statistics: A Vector Space Approach, John Wiley \& Sons, New York, 1983.

${ }^{27}$ Girko, V. L., Theory of Random Determinants, Kluwer Academic Publishers, The Neatherlands, 1990.

${ }^{28}$ Muirhead, R. J., Aspects of Multivariate Statistical Theory, John Wiely and Sons, New York, USA, 1982.

${ }^{29}$ Mehta, M. L., Random Matrices, Academic Press, San Diego, CA, 2nd ed., 1991.

${ }^{30}$ Gupta, A. and Nagar, D., Matrix Variate Distributions, Monographs \& Surveys in Pure \& Applied Mathematics, Chapman \& Hall/CRC, London, 2000.

${ }^{31}$ Janik, R. A. and Nowak, M. A., "Wishart and anti-Wishart random matrices," Journal of Physics A: Mathematical and General, Vol. 36, No. 2, 2003, pp. 3629-3637.

${ }^{32}$ Langley, R. S. and Brown, A. W. M., "The ensemble statistics of the energy of a random system subjected to harmonic excitation," Journal of Sound and Vibration, Vol. 275, August 2004, pp. 823-846.

${ }^{33}$ Adhikari, S. and Friswell, M. I., "Random matrix eigenvalue problems in structural dynamics," International Journal for Numerical Methods in Engineering, Vol. 69, No. 3, 2007, pp. 562-591.

${ }^{34}$ Caughey, T. K. and O'Kelly, M. E. J., "Classical normal modes in damped linear dynamic systems," Transactions of ASME, Journal of Applied Mechanics, Vol. 32, September 1965, pp. 583-588.

${ }^{35}$ Adhikari, S., "Damping modelling using generalized proportional damping," Journal of Sound and Vibration, Vol. 293, No. 1-2, May 2006, pp. 156-170, (among the top 25 most downloaded articles in January - March 2006). 